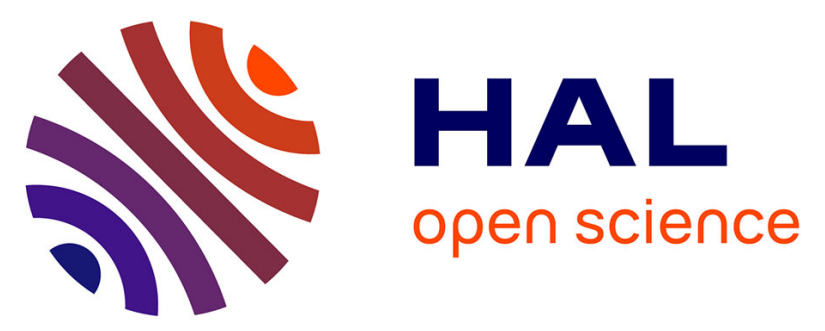

\title{
Evaluation of the ability of linezolid and tedizolid to eradicate intraosteoblastic and biofilm-embedded Staphylococcus aureus in the bone and joint infection setting
}

Lélia Abad, Virginie Tafani, Jason Tasse, Jérôme Josse, Christian Chidiac, Sebastien Lustig, Tristan Ferry, Alan Diot, Frédéric Laurent, Florent Valour

\section{To cite this version:}

Lélia Abad, Virginie Tafani, Jason Tasse, Jérôme Josse, Christian Chidiac, et al.. Evaluation of the ability of linezolid and tedizolid to eradicate intraosteoblastic and biofilm-embedded Staphylococcus aureus in the bone and joint infection setting. Journal of Antimicrobial Chemotherapy, 2019, 74 (3), pp. 625-632. 10.1093/jac/dky473 . hal-02074564

\author{
HAL Id: hal-02074564 \\ https://hal.science/hal-02074564
}

Submitted on 20 May 2021

HAL is a multi-disciplinary open access archive for the deposit and dissemination of scientific research documents, whether they are published or not. The documents may come from teaching and research institutions in France or abroad, or from public or private research centers.
L'archive ouverte pluridisciplinaire HAL, est destinée au dépôt et à la diffusion de documents scientifiques de niveau recherche, publiés ou non, émanant des établissements d'enseignement et de recherche français ou étrangers, des laboratoires publics ou privés. 


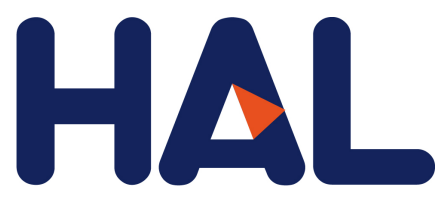

archives-ouvertes

\section{Evaluation of the ability of linezolid and tedizolid to eradicate intraosteoblastic and biofilm-embedded Staphylococcus aureus in the bone and joint infection setting}

Lélia Abad, Virginie Tafani, Jason Tasse, Jérôme Josse, Christian Chidiac, Sebastien Lustig, Tristan Ferry, Alan Diot, Frédéric Laurent, Florent Valour

\section{To cite this version:}

Lélia Abad, Virginie Tafani, Jason Tasse, Jérôme Josse, Christian Chidiac, et al.. Evaluation of the ability of linezolid and tedizolid to eradicate intraosteoblastic and biofilm-embedded Staphylococcus aureus in the bone and joint infection setting. Journal of Antimicrobial Chemotherapy, Oxford University Press (OUP), 2019, 74 (3), pp. 625-632. 10.1093/jac/dky473 . hal-02074564

\section{HAL Id: hal-02074564 \\ https://hal.archives-ouvertes.fr/hal-02074564}

Submitted on 20 May 2021

HAL is a multi-disciplinary open access archive for the deposit and dissemination of scientific research documents, whether they are published or not. The documents may come from teaching and research institutions in France or abroad, or from public or private research centers.
L'archive ouverte pluridisciplinaire HAL, est destinée au dépôt et à la diffusion de documents scientifiques de niveau recherche, publiés ou non, émanant des établissements d'enseignement et de recherche français ou étrangers, des laboratoires publics ou privés. 


\title{
Evaluation of the ability of linezolid and tedizolid to eradicate intraosteoblastic and biofilm-embedded Staphylococcus aureus in the bone and joint infection setting
}

\author{
Lélia Abad $^{1-4}$, Virginie Tafani ${ }^{1}$, Jason Tasse ${ }^{1}$, Jérôme Josse ${ }^{1}$, Christian Chidiac ${ }^{1-3,5}$, Sébastien Lustig $^{2,3,6}$, \\ Tristan Ferry $^{1-3,5}$, Alan Diot ${ }^{1}$, Frédéric Laurent ${ }^{1-4, *} \dagger$ and Florent Valour ${ }^{1-3,5} \dagger$ \\ ${ }^{1}$ CIRI - Centre International de Recherche en Infectiologie, Inserm, U1111, Université Claude Bernard Lyon 1, CNRS, UMR5308, Ecole \\ Normale Supérieure de Lyon, Université Lyon, F-69007, Lyon, France; ${ }^{2}$ Regional Reference Centre for Complex Bone and Joint Infection \\ (CRIOAc Lyon), Hospices Civils de Lyon, Lyon, France; ${ }^{3}$ Université Claude Bernard Lyon 1, Lyon, France; ${ }^{4}$ Department of Bacteriology, \\ Institute for Infectious Agents, Hospices Civils de Lyon, Lyon, France; ${ }^{5}$ Department of Infectious Diseases, Hospices Civils de Lyon, \\ Lyon, France; ${ }^{6}$ Department of Orthopaedic Surgery, Hospices Civils de Lyon, Lyon, France
}

\begin{abstract}
*Corresponding author. Tel: +33-4-72-07-18-39; Fax: +33-4-72-07-17-50; E-mail: Frederic.laurent@univ-lyon1.fr
†These authors contributed equally to this work.
\end{abstract}

Received 19 July 2018; returned 5 September 2018; revised 8 October 2018; accepted 16 October 2018

\begin{abstract}
Objectives: Prolonged use of linezolid for bone and joint infection (BJI) is limited by its long-term toxicity. The better safety profile of tedizolid, a recently developed oxazolidinone, could offer an alternative. However, its efficacy against biofilm-embedded and intracellular Staphylococcus aureus, the two main bacterial reservoirs associated with BJI chronicity, is unknown.
\end{abstract}

\begin{abstract}
Methods: Using three S. aureus strains (6850 and two clinical BJI isolates), linezolid and tedizolid were compared regarding their ability: (i) to target the $S$. aureus intracellular reservoir in an in vitro model of osteoblast infection, using three concentrations increasing from the bone concentration reached with standard therapeutic doses $\left(C_{\text {bone }}=2.5 \times\right.$ MIC; $C_{\text {plasm }}=10 \times$ MIC; $C_{\max }=40 \times$ MIC); (ii) to eradicate mature biofilm [minimal biofilm eradication concentration (MBEC)]; and (iii) to prevent biofilm formation [biofilm MIC (bMIC) and confocal microscopy].
\end{abstract}

\begin{abstract}
Results: Linezolid and tedizolid weakly reduced the intracellular inoculum of S. aureus in a strain-dependent manner despite the similar MICs for the tested strains, but improved cell viability even in the absence of an intracellular bactericidal effect. Conversely, linezolid and tedizolid were ineffective in eradicating mature biofilm formed in vitro, with MBEC $>2000$ and $>675 \mathrm{mg} / \mathrm{L}$, respectively. bMICs of tedizolid were 4 -fold lower than those of linezolid for all strains.

Conclusions: Linezolid and tedizolid alone are not optimal candidates to target bacterial phenotypes associated with chronic forms of BJI. Despite weak intracellular activity, they both reduce infection-related cytotoxicity, suggesting a role in modulating intracellular expression of staphylococcal virulence factors. Although inactive against biofilm-embedded S. aureus, both—but particularly tedizolid—are able to prevent biofilm formation.
\end{abstract}

\section{Introduction}

The leading cause of bone and joint infection (BJI), Staphylococcus aureus, is responsible for difficult-to-treat infections because of its wide panel of virulence factors, which allows host colonization, tissue invasion and subversion of the host immune response. ${ }^{1-3}$ Chronic and recurrent forms, frequently observed in staphylococcal BJI, have been associated with specific phenotypic mechanisms responsible for subsequent emergence of bacterial reservoirs. First, biofilm formation has been related to persistent BJIs, protecting the pathogen from the extracellular host defences and most antimicrobials. ${ }^{4}$ Consequently, molecules able to diffuse and remain active against $S$. aureus in biofilms, such as rifampicin, have been highlighted in current guidelines for playing a pivotal role in the management of BJI, especially in cases of orthopaedic device-related infections., 5 Second, several studies have linked the ability of $S$. aureus to invade and persist within nonprofessional phagocytic bone cells, such as osteoblasts, with BJI chronicity. 7,8 The intraosteoblastic activity of antistaphylococcal drugs has not been extensively evaluated so far and is consequently not taken into consideration in the choice of current therapeutic strategies, although it may be a key player in relapse prevention and treatment outcome. ${ }^{9}$ 
While rifampicin and ofloxacin present the optimal intracellular and antibiofilm activity profile, ${ }^{6,9}$ they can be associated with several side effects and drug interactions, ${ }^{10}$ and select for bacterial resistance. ${ }^{11,12}$ Linezolid, an oxazolidinone antibiotic, is increasingly used in the treatment of BJI, because of its $100 \%$ oral bioavailability and acceptable bone and joint tissue penetration. ${ }^{13,14}$ Oxazolidinones bind to the $23 \mathrm{~S}$ ribosomal RNA, thus inhibiting protein synthesis and conferring bacteriostatic activity against a range of Gram-positive bacteria. ${ }^{15}$ However, its off-label prolonged use required for $\mathrm{BJI}$ exposes patients to frequent haematological and neurological adverse events, due to secondary inhibition of mitochondrial protein synthesis. ${ }^{16-18}$ Moreover, resistance to linezolid, mediated by chromosomal mutations in $23 \mathrm{~S}$ ribosomal RNA or, more rarely, horizontal gene acquisition (cfr, optrA, poxtA) is increasing in S. aureus. ${ }^{19}$ In this context, tedizolid (formally TR-700 or torezolid), a second-generation oxazolidinone, has recently been approved for the treatment of acute skin and skin structure infections. This drug shows bacteriostatic activity against most of the Gram-positive species responsible for BJI. ${ }^{15,20}$ Although $23 \mathrm{~S}$ ribosomal RNA mutations cause cross-resistance between linezolid and tedizolid, tedizolid retains activity against linezolid-resistant cfr-positive strains because of its hydroxymethyl group. ${ }^{21-23}$ Moreover, the lower daily dose of tedizolid $(200 \mathrm{mg})$ compared with linezolid $(1200 \mathrm{mg})$ gives hopes for a better long-term tolerance profile, because of significantly reduced mitochondrial drug exposure. ${ }^{24,25}$ Therefore, tedizolid can be considered for prolonged used in patients with BJI.

However, one must take into account the lack of data regarding the ability of the two oxazolidinones to eradicate biofilmembedded S. aureus and the intracellular reservoir associated with BJI chronicity. In this context, the aim of this study was to assess the activity of linezolid and tedizolid against $S$. aureus in in vitro models of osteoblast infection and biofilm formation.

\section{Materials and methods}

\section{Bacterial strains}

All experiments were performed using three MSSA strains: the reference strain 6850 and two clinical isolates (named clinical isolates 1 and 2) collected from patients with recurrent osteomyelitis. MICs of linezolid and tedizolid were determined by the Etest method, according to the manufacturer's instructions (bioMérieux, Marcy-l'Étoile, France) and the recommendation of the French Committee for Antimicrobial Susceptibility Testing (CA-SFM).

\section{Intracellular activity of oxazolidinones and impact on infection-induced cytotoxicity}

Intracellular activity of linezolid (Pfizer, New York, USA) and tedizolid (Ark Pharm, Arlington Heights, USA) was assessed in an in vitro model of osteoblast infection, as previously described. ${ }^{9}$

The human osteoblastic cell line MG63 (CRL-1427; LGC Standards, $U S A)^{26}$ was routinely cultured in growth medium DMEM (Gibco ${ }^{\text {TM }}$, Paisley, UK) supplemented with $10 \%$ FCS (Gibco $\left.{ }^{\mathrm{TM}}\right), \pm 100 \mathrm{U} / \mathrm{mL}$ penicillin and $100 \mathrm{mg} / \mathrm{L}$ streptomycin (Gibco ${ }^{\mathrm{TM}}$ ), in a humidified incubator at $37^{\circ} \mathrm{C}$ in a $5 \%$ $\mathrm{CO}_{2}$ atmosphere. Cells were passaged once a week and used up to passage 20 after thawing. Prior to assays, osteoblasts were seeded at 50000 cells per well into 24-well tissue culture plates (Falcon, Le Pont de Claix, France) in $1 \mathrm{~mL}$ of growth medium with antibiotics and cultured for $48 \mathrm{~h}$ until $70 \%$ $80 \%$ confluence.
Before each experiment, tested strains were subcultured on Columbia agar supplemented with $5 \%$ sheep blood (COS; bioMérieux) at $37^{\circ} \mathrm{C}$ for $24 \mathrm{~h}$. Three colonies of each strain were then used to inoculate brain heart infusions (BHIs; bioMérieux), incubated overnight at $37^{\circ} \mathrm{C}$. Bacterial suspensions were then centrifuged at $3000 \mathrm{rpm}$ and resuspended in cell culture medium without antibiotics. An aliquot was spiral-plated on COS for bacterial enumeration, using an automated plater (Easyspira ${ }^{\circledR}$ Interscience, Saint-Nom-la-Bretèche, France). Suspensions were kept overnight at $4^{\circ} \mathrm{C}$. The next day, bacterial suspensions were standardized at an moi of 100 bacteria/cell and added to the bone cell culture wells for $2 \mathrm{~h}$ at $37^{\circ} \mathrm{C}$ to allow bacterial internalization in osteoblasts. Cells were then washed twice with PBS (Gibco ${ }^{T M}$ ) and incubated for $1 \mathrm{~h}$ with growth medium supplemented with $10 \mathrm{mg} / \mathrm{L}$ lysostaphin (Sigma-Aldrich, St Louis, MO, USA) to kill the remaining extracellular bacteria. Infected cells were then washed twice with PBS and incubated for $24 \mathrm{~h}$ with growth medium containing the tested antimicrobials.

A range of three concentrations was used to assess potential dose effects and were chosen on the basis of both the MICs for the tested strains and clinically relevant concentrations determined after a pharmacological literature review: ${ }^{14,27}$ (i) the lower concentrations were set at the intraosseous concentration reached in humans when using standard therapeutic dosages $\left(C_{\text {bone }}\right)$, corresponding to $2.5 \times$ MIC for the three isolates; (ii) the medium concentration was set at $10 \times \mathrm{MIC}$, corresponding to the human plasma concentration ( $\left.C_{\text {plasm }}\right)$; and (iii) a maximal concentration was set at $40 \times \operatorname{MIC}\left(C_{\text {max }}\right)$ to assess a possible dose effect.

For each condition, lysostaphin at $10 \mu \mathrm{g} / \mathrm{mL}$ was also added to the growth medium to rapidly kill the extracellular bacteria released upon host cell lysis. After $24 \mathrm{~h}$ of incubation, osteoblasts were washed twice with PBS and subsequently lysed by a 10 min incubation with $1 \mathrm{~mL}$ of sterile water. Serial dilutions of these lysates were spiral-plated in duplicate on COS for enumeration of intracellular bacteria (Easyspira ${ }^{\circledR}$ Interscience).

A colorimetric assay based on the enzymatic reduction of MTT to MTTformazan was used to determine the viability of: (i) uninfected cells exposed to the antimicrobials at the different tested concentrations in order to assess a possible drug-induced cytotoxic effect; and (ii) untreated and treated infected cells to evaluate the impact of treatment on the cytotoxicity induced by infection. Briefly, MTT reagent (Sigma-Aldrich) was dissolved in sterile water $(5 \mathrm{mg} / \mathrm{mL})$. Culture medium was eliminated and wells were washed twice with PBS. Then $400 \mu \mathrm{L}$ of fresh DMEM $+10 \%$ FBS with diluted MTT $(1 / 10,10 \% \mathrm{MTT})$ was added. After $1 \mathrm{~h}$ of incubation at $37^{\circ} \mathrm{C}$, this medium was removed and formazan crystals were dissolved in $200 \mu \mathrm{L}$ of acidified isopropanol. MTT reduction was quantified by measuring the light absorbance at $450 \mathrm{~nm}$ using a microplate reader (Tecan Infinite ${ }^{\circledR}$ 200 PRO, Männedorf, Switzerland).

\section{Prevention of biofilm formation}

The ability of linezolid and tedizolid to prevent biofilm formation was assessed by determination of their biofilm MICs (bMICs) using the Antibiofilmogram ${ }^{\circledR}$ test (BioFilm Control, Saint Beauzire, France), as previously described. ${ }^{28}$ Briefly, overnight cultures in BHI were standardized to an $\mathrm{OD}_{600}$ of $1 \pm 0.05$ (Ultrospec 10 Cell Density Meter, Amersham Biosciences, USA) and diluted in sterile BHI to obtain a final concentration of $6 \times 10^{6} \mathrm{cfu} / \mathrm{mL}$. These bacterial suspensions were mixed with $1 \%$ of 'Toner', consisting of a magnetic bead suspension and seeded in a 96-well microplate ( $200 \mu \mathrm{L} /$ well). Twenty microlitres of antibiotic solutions, in a range of eight 2-fold dilutions from 16 to $0.0156 \mathrm{mg} / \mathrm{L}$, was added to each well. Microplates were incubated at $37^{\circ} \mathrm{C}$ for $4 \mathrm{~h}$. Opac oil (contrast liquid) was deposited on the surface of each well ( $100 \mu \mathrm{L} /$ well) and plates were placed for $1 \mathrm{~min}$ on a dedicated block for magnetization before the bottom of each well was scanned with a specific plate reader (Pack BIOFILM, Biofilm Control); free beads were attracted to the centre of the well to form a bright spot. Its intensity decreased as the beads were immobilized by biofilm formation. The bMIC of each antibiotic corresponded to the 
lowest concentration for which a spot, similar to negative control without bacteria, was visible.

\section{Eradication of biofilm-embedded S. aureus}

The ability of linezolid and tedizolid to eradicate $S$. aureus embedded in mature biofilm was assessed by determining their minimum biofilm eradication concentration (MBEC). The MBEC corresponds to the lowest concentration of antimicrobial that eradicates $99.9 \%$ of the bacteria embedded in a biofilm compared with control in the same conditions without antimicrobial. ${ }^{29}$ Suspensions were standardized to an $\mathrm{OD}_{600}$ of $1 \pm 0.05$ and diluted 1/100 in fresh BHI 1\% glucose (237500, BD, Germany). A 96well polystyrene plate (Greiner Bio-One, Kremsmünster, Austria) was then inoculated with $200 \mu \mathrm{L}$ of bacterial suspension per well and incubated at $37^{\circ} \mathrm{C}$ for $48 \mathrm{~h}$. The supernatant was removed and biofilms were carefully washed with steam. Biofilms were treated with $0-2000 \mathrm{mg} / \mathrm{L}$ linezolid or 0-675 mg/L tedizolid in $180 \mu \mathrm{L}$ of Mueller-Hinton broth for $48 \mathrm{~h}$ at $37^{\circ} \mathrm{C}$. Supernatant was then removed and biofilms were washed before being resuspended in $200 \mu \mathrm{L}$ of PBS by scraping the wells with sterile pipette tips and 10 min of sonication. Bacterial counts were assessed by plating serial dilutions onto COS. The MBEC was defined as the first concentration at which no colony growth was observed.

\section{Confocal laser scanning microscopy (CLSM)}

The efficiency of linezolid and tedizolid in preventing biofilm formation was visually assessed by CLSM performed on a 4-h-old biofilm for all strains. Briefly, biofilm was formed on eight-well uncoated microslides (iBidi ${ }^{\circledR}$; Martinsried, Germany) as described above and fluorescently stained with Syto9 and propidium iodide from the Live/Dead ${ }^{\circledR}$ BacLight $^{\text {TM }}$ Bacterial Viability Kit (Molecular Probes ${ }^{\mathrm{TM}}$, Eugene, USA), according to the manufacturer's instructions. Biofilms were then incubated for $30 \mathrm{~min}$ at room temperature in the dark before being washed. Biofilms were observed using a $\times 20$ dry lens (Plan-Apochromat 20×10.8 M27; Leica Microsystems, Wetzlar, Germany). Images were acquired in a Zeiss LSM800, Full GaAsP detectors (Leica Microsystems) CLSM, at $2524 \times 2524$ resolution in at least three different areas of each analysed surface, and 3D reconstructions were performed using Imaris (version 8.0) software (Bitplane, Belfast, UK). Biofilm quantification was performed using Comstat2 (University of Denmark). ${ }^{30}$

\section{Statistical methods}

Three independent experiments were performed in triplicate. Data were summarized as means of these nine measurement points and their 95\% CIs. Normal distribution was not assumed, so variables were compared using a non-parametric two-sided Mann-Whitney $U$-test. Dose effect was assessed by linear regression between $C_{\text {bone, }} C_{\text {plasm }}$ and $C_{\max }$. A $P$ value $<0.05$ was considered significant. All analyses were performed using Prism software (GraphPad, San Diego, USA).

\section{Results}

\section{Susceptibility studies}

The three $S$. aureus strains (6850 and clinical isolates 1 and 2) had the same MICs of linezolid $(1.5 \mathrm{mg} / \mathrm{L})$ and tedizolid $(0.25 \mathrm{mg} / \mathrm{L})$, and were consequently fully susceptible to both oxazolidinones.

\section{Linezolid and tedizolid cytotoxicity to uninfected cells}

MTT assays performed after treatment of uninfected cells with linezolid and tedizolid at $C_{\text {bone, }}, C_{\text {plasm }}$ and $C_{\max }$ demonstrated that the antimicrobials had no significant impact on cell viability in this range of concentrations (Figure S1, available as Supplementary data at JAC Online).

\section{Intraosteoblastic activity of linezolid and tedizolid and impact on infection-induced cytotoxicity}

Only linezolid was able to significantly decrease $S$. aureus 6850 strain intracellular inoculum at $C_{\text {plasm }}$ and $C_{\text {max }}$, with a significant dose response $[-22.0 \%(95 \% \mathrm{CI},-33.0 \%$ to $-11.0 \% ; P=0.012)$ and $-31.1 \%$ ( $95 \% \mathrm{CI},-40.8 \%$ to $-21.4 \% ; P=0.002)$, respectively]. No significant change in the intracellular amount of $S$. aureus 6850 was observed after treatment with tedizolid (Figure 1a). Both molecules significantly improved the viability of infected cells at all tested concentrations, regardless of the existence of an intracellular bactericidal effect (Figure 1b).

Regarding clinical isolate 1 , a reduction of intracellular bacteria was observed for both linezolid and tedizolid at all tested concentrations (Figure 1c) even at $C_{\text {bone }}$, at which $-54.3 \%(95 \%$ CI, $-64.9 \%$ to $-43.8 \% ; P<0.0001)$ and $-55.6 \%(95 \% \mathrm{CI},-61.75 \%$ to $-49.5 \%$; $P<0.0001$ ) of intracellular bacteria were observed compared with untreated controls, respectively. A significant concentration-dependent effect was observed for linezolid and tedizolid. Infection-induced cytotoxicity was reduced by both molecules at all concentrations compared with untreated cells (Figure 1d).

Finally, linezolid and tedizolid both significantly reduced the intracellular inoculum of osteoblasts infected by clinical isolate 2 from $C_{\text {bone }}$ by $39.3 \%$ (95\% CI, 27.6\%-51.05\%; $P=0.0002$ ) and $45.2 \%(95 \% \mathrm{CI}, 31.9 \%-58.5 \% ; P=0.0001)$, respectively (Figure 1e), with a significant increase in cell viability observed only for linezolid at $C_{\max }$ (Figure 1f).

\section{Ability to prevent biofilm formation}

Linezolid and tedizolid bMICs defined using the Antibiofilmogram ${ }^{\circledR}$ method are given in Figure 2(a). Both molecules were able to prevent biofilm formation by all $S$. aureus isolates, with bMICs being 4fold lower for tedizolid than for linezolid.

CLSM analysis of the proportion of biofilm-embedded viable cells after treatment with linezolid and tedizolid at bMICs showed a significant reduction of viable bacteria with tedizolid compared with untreated cells for the three strains (Figure $2 b$ and $c$ ). The reduction of viable cells observed after treatment with linezolid reached statistical significance for the two clinical strains, but not for strain 6850 .

\section{Ability to eradicate mature biofilm}

No significant change was observed regarding bacterial inoculum embedded in mature biofilm after addition of increasing concentrations of the two antibiotics. Linezolid and tedizolid MBECs were $>2000$ and $>675 \mathrm{mg} / \mathrm{L}$ for the three tested isolates (Figure 3).

\section{Discussion}

S. aureus BJI represents a severe clinical issue, frequently evolving into chronic and treatment-refractory forms despite appropriate surgical management and prolonged antimicrobial therapy. In the BJI setting, unlike most other acute bacterial infections, in vitro antimicrobial susceptibility of clinical isolates appears insufficient to predict a favourable outcome. Indeed, the eradication of a fully susceptible isolate can be impaired by bacterial phenotypes, allowing the subversion of the host immune system and of the action of antimicrobials, including intracellular and biofilm-associated 
(a)

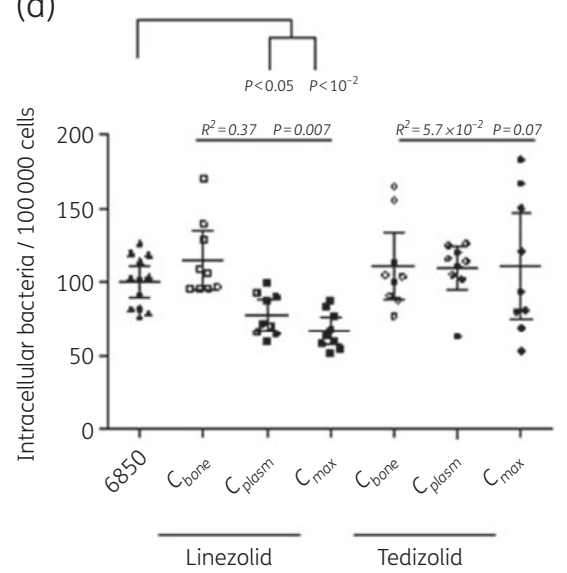

(b)

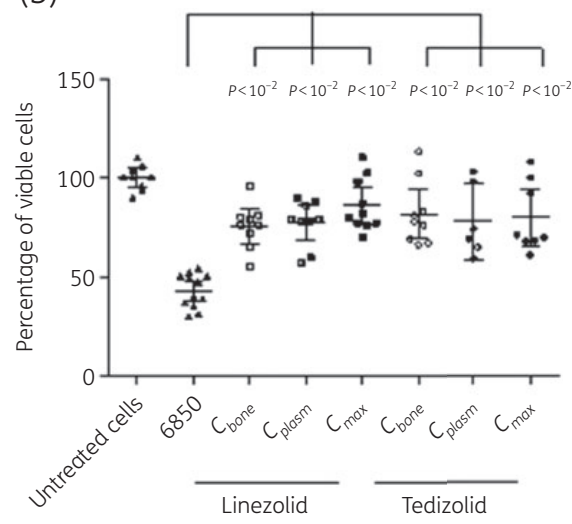

(c)

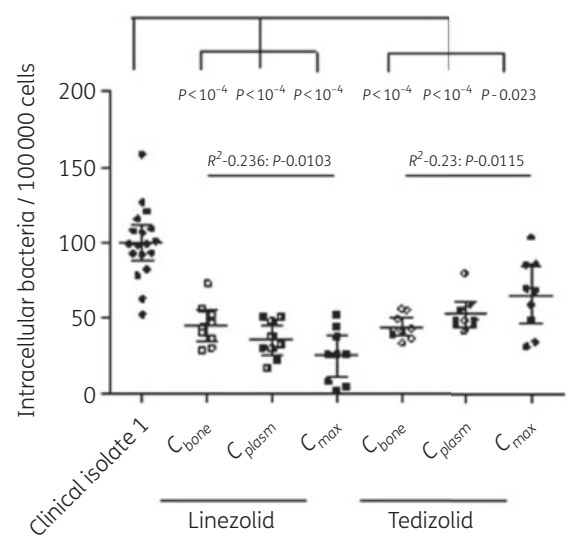

(d)

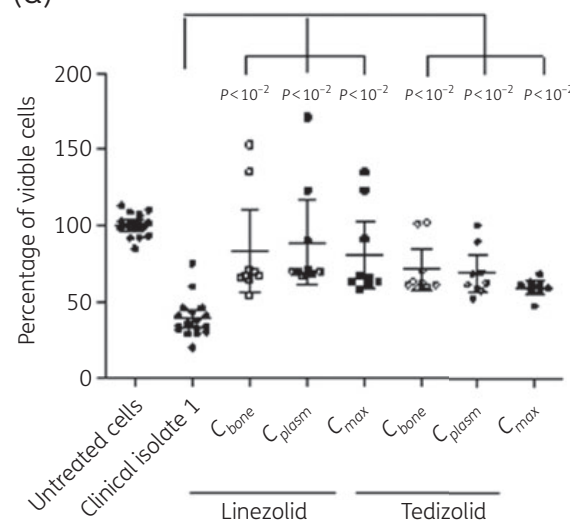

(e)

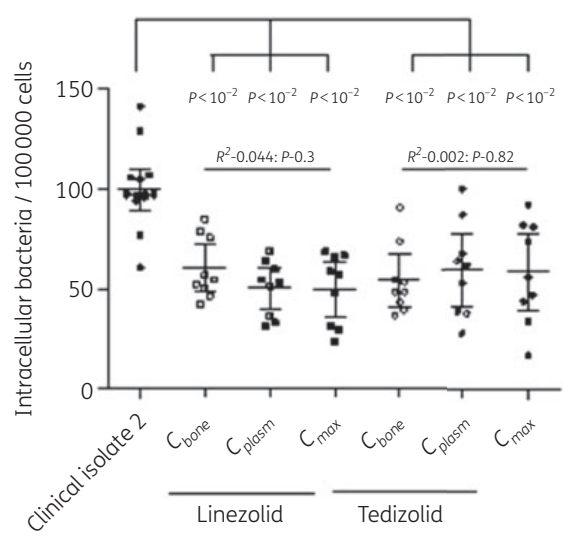

(f)

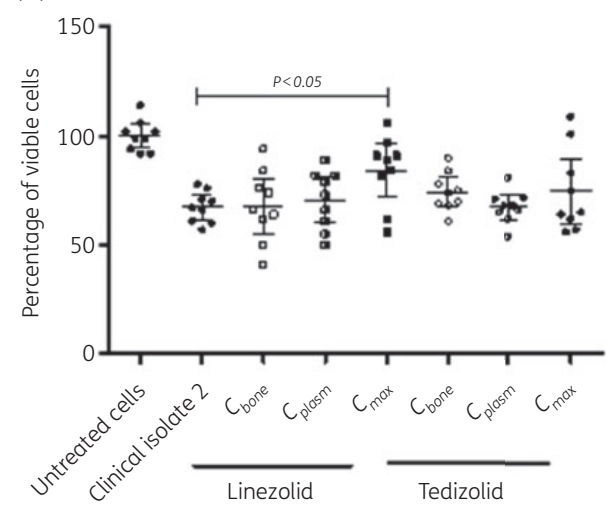

Figure 1. Ability of linezolid and tedizolid to eradicate intraosteoblastic $S$. aureus ( $a, c$ and e) and prevent infection-induced cytotoxicity (b, d and f) using one reference strain (S. aureus 6850) and two BJI clinical isolates. Three concentrations were used: the bone concentration reached in humans under standard therapeutic conditions $\left(C_{\text {bone }}=2.5 \times \mathrm{MIC}\right)$, the plasma concentration $\left(C_{\text {plasm }}=10 \times \mathrm{MIC}\right)$ and a maximal concentration $\left(C_{\text {max }}=40 \times\right.$ MIC). Differences between treatment doses were assessed using the Mann-Whitney U-test, and the dose effect was assessed using linear regression.

lifestyles. ${ }^{4,7,8,31,32}$ Consequently, evaluation of the ability of antimicrobials to target these bacterial reservoirs associated with BJI chronicity is likely pivotal for the choice of treatment strategies aiming at reducing the risk of infection recurrence and chronicization.

To the best of our knowledge, the present study represents the first comparison of linezolid and tedizolid efficacy against these persistence mechanisms and was designed to mimic as closely as possible the relevant clinical conditions encountered in the BJI setting by using: (i) three different strains, including one reference strain (6850) and two clinical isolates implicated in chronic BJIs; (ii) a range of concentrations chosen according to both the MICs for the tested strains and human concentrations reached with standard therapeutic dosing in plasma and bone tissue; (iii) an osteoblastic cell infection model, which appears more relevant than the macrophage/monocyte models commonly used to assess the intracellular activity of antistaphylococcal molecules when aiming to specifically target mechanisms associated with BJI chronicity; and (iv) biofilm models that allow the evaluation of prevention and eradicative activities of oxazolidinones.
Our results showed no drug-induced cytotoxicity, regardless of the antibiotic concentrations used, making the intracellular results interpretable. Oxazolidinones showed strain-dependent intracellular activity, despite the similar MICs of each antibiotic for the three tested strains. Although a reduction of the intracellular reservoir of the $S$. aureus 6850 reference strain was only observed with linezolid at $C_{\text {plasm }}$, linezolid and tedizolid were able to decrease the intracellular bacterial load by at least $30 \%$ in osteoblasts infected by the two clinical isolates at the lowest concentration used $\left(C_{\text {bone }}\right)$. In a preliminary study using linezolid at bone concentration, our group showed a higher decrease in intraosteoblastic S. aureus HG001 load than observed in the present study; this is likely related to inter-strain variability. ${ }^{9}$ Mélard et $a .^{33}$ showed a $0.26 \log _{10}$ decrease in intracellular cfu in TPH-1 monocytes but the cellular model was different and a higher concentration of linezolid (17.5 mg/L) was used. Moreover, a paradoxical effect of tedizolid on clinical isolate 1 was observed, with an unexplained increase in the number of intracellular bacteria at $C_{\max }$ compared with the lower concentrations. As MICs for the three strains were similar, this inter-strain variability suggests that phenotypic modifications occur after internalization, leading to intracellular antibiotic 
(a)

\begin{tabular}{lcc}
\hline bMIC $(\mathrm{mg} / \mathrm{L})$ & Linezolid & Tedizolid \\
\hline $\begin{array}{l}\text { 6850 reference } \\
\text { strain }\end{array}$ & 1 & 0.25 \\
Clinical isolate 1 & 1 & 0.25 \\
Clinical isolate 2 & 2 & 0.5 \\
\hline
\end{tabular}

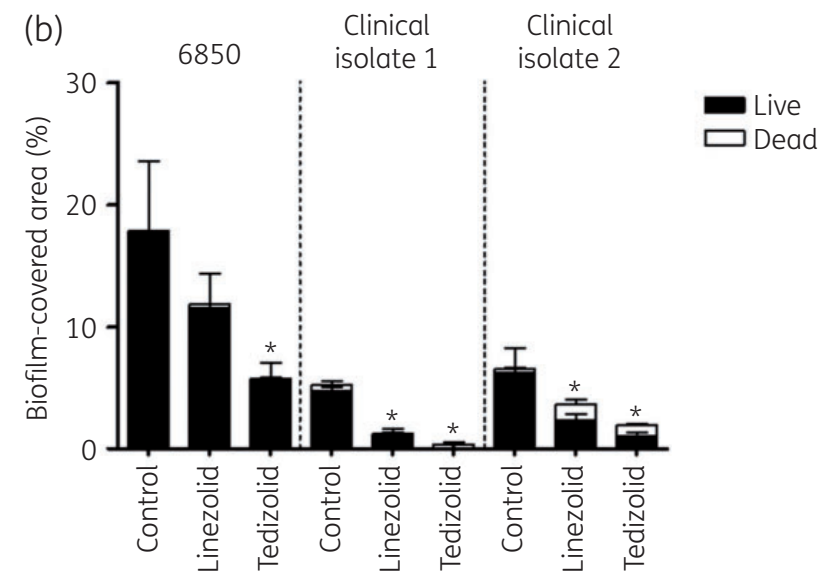

Linezolid

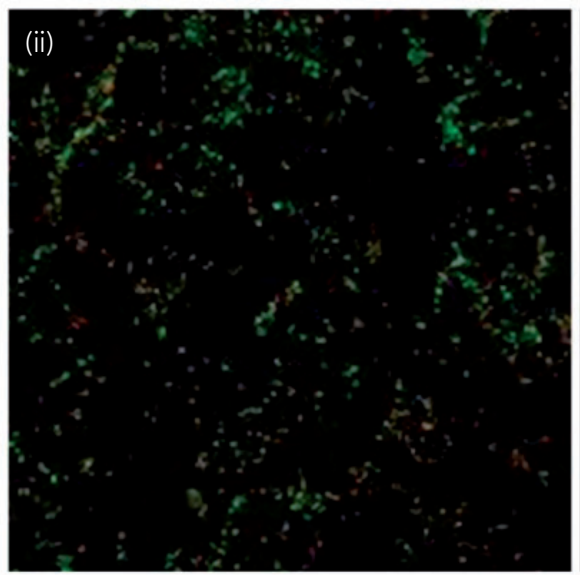

Tedizolid

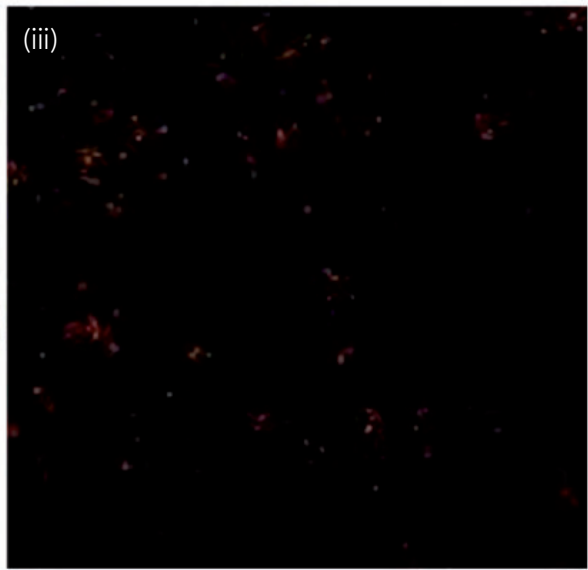

Figure 2. Ability of linezolid and tedizolid to prevent biofilm formation. (a) bMICs of linezolid and tedizolid for the S. aureus 6850 reference strain and two BJI clinical isolates determined using the Antibiofilmogram ${ }^{\circledR}$ method. (b) Proportion of S. aureus viable cells determined using CLSM after treatment with linezolid or tedizolid at bMICs compared with untreated cells. (c) CLSM showing biofilm-embedded viable (green) and dead (red) S. aureus 6850 without treatment (i), after treatment with linezolid (ii) and after treatment with tedizolid (iii). This figure appears in colour in the online version of JAC and in black and white in the print version of JAC.

susceptibility changes. Several hypotheses can be raised: (i) variability of intracellular emergence of small colony variants (SCVs), which are usually less susceptible to antimicrobials than their parental strain; ${ }^{7,34}$ (ii) strain-dependent bacterial cell wall modifications, which have been shown to decrease intracellular antimicrobial susceptibility; ${ }^{35}$ and/or (iii) differential location of intracellular sanctuarization (vacuole, cytosol or lysosome) depending on the virulence factors of the strains, thus modifying local environmental parameters, especially $\mathrm{pH}$, which is known to greatly impact antimicrobial efficacy. ${ }^{36,37}$ There are no published studies in which the subcellular location of linezolid or tedizolid was examined. However, radezolid, another oxazolidinone, has been described to be mainly localized in lysosomes in human TPH1 cells. $^{38}$

Another interesting point is the ability of linezolid and tedizolid to reduce infection-induced cytotoxicity, even in experimental conditions where no reduction of the intracellular bacterial counts was observed. As oxazolidinones target the bacterial protein synthesis process, this phenomenon is likely explained by a decrease in bacterial secretion of cytolytic compounds such as toxins due to antimicrobial effect. ${ }^{39}$ Hence, phenol-soluble modulins (PSMs) could be key players in this hypothesis, because: (i) their secretion by intraosteoblastic $S$. aureus and their role in infection-induced cytotoxicity have been previously demonstrated in our laboratory; ${ }^{40}$ and (ii) both linezolid and tedizolid have been reported to be able to reduce PSM secretion in a strain-dependent manner and under specific concentration conditions. ${ }^{39,41,42}$

The Antibiofilmogram ${ }^{\circledR}$ method and confocal microscopy experiments showed the efficiency of the two oxazolidinones in preventing biofilm formation for all the tested strains. Of note, tedizolid presented lower bMICs than linezolid. Interestingly, S. aureus 6850 and clinical isolate 1 displayed lower bMICs than MICs. This result is likely due to the mechanism of action of oxazolidinones, which targets ribosomes. It is likely that these antibiotics (known as anti-toxin antibiotics) are able to inhibit the production of proteins (as already described for staphylococcal toxins ${ }^{43}$ ) involved in bacterial adhesion (first step of biofilm formation), without killing bacteria. This phenomenon could explain why bMIC is actually lower than MIC for strains 6850 and clinical isolate 1 . Consequently, these results suggest the potential interest of the 


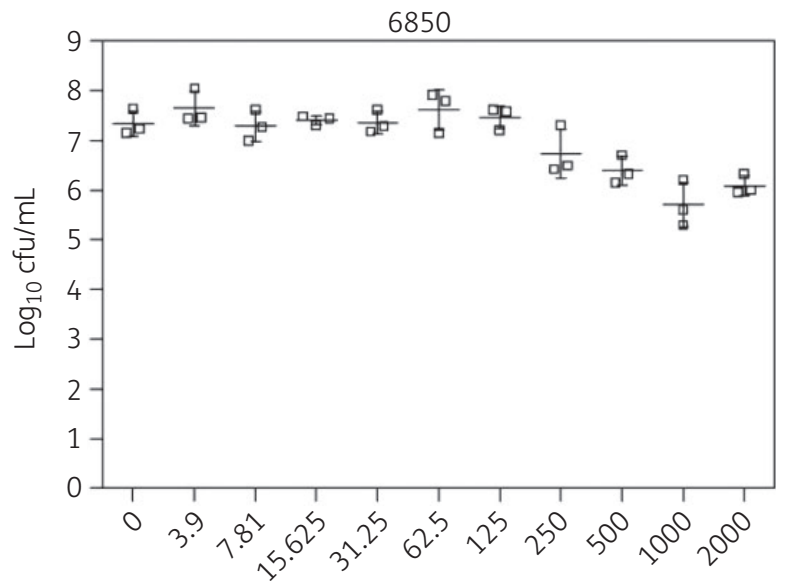

Linezolid concentration (mg/L)

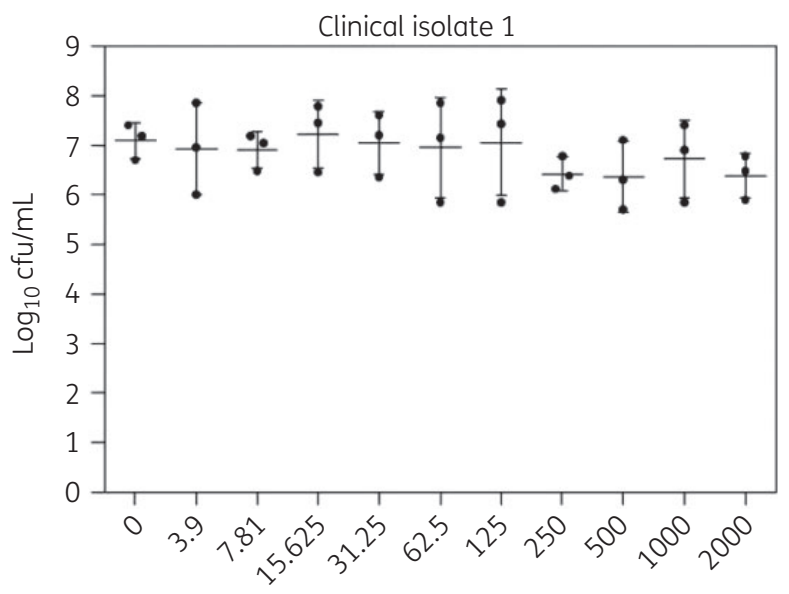

Linezolid concentration (mg/L)

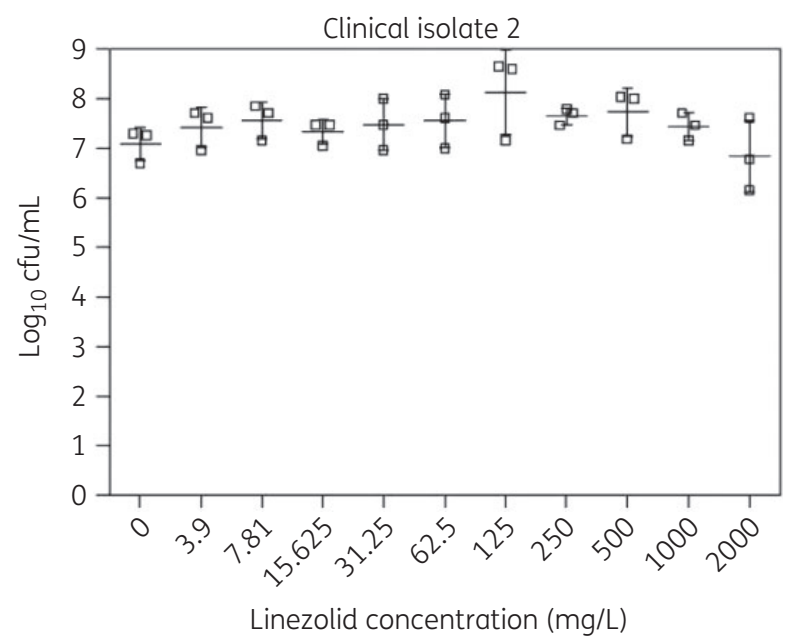

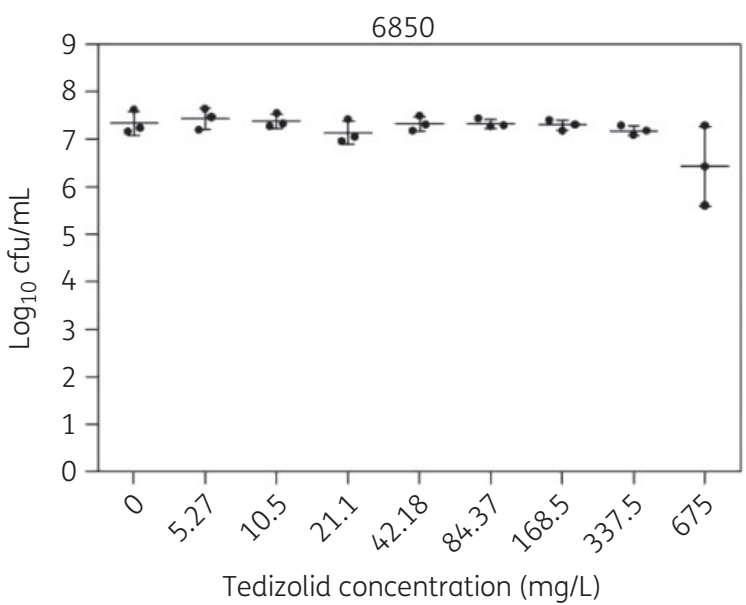
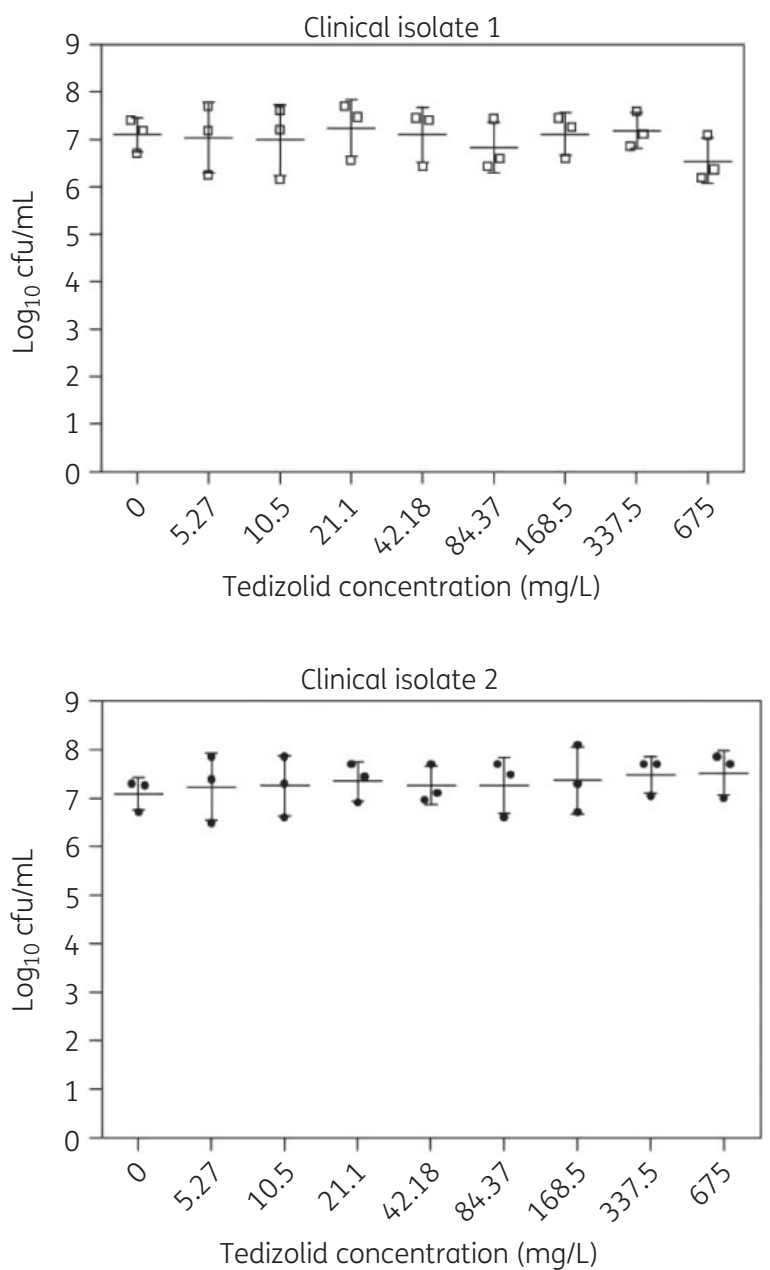

Figure 3. Determination of the MBECs of linezolid and tedizolid for S. aureus reference strain 6850 and two BJI clinical isolates.

evaluation of oxazolidinones, especially tedizolid, as systemic and/ or local (antibiotic-loaded cements or coated orthopaedic implants) antimicrobial prophylaxis to prevent biofilm-associated infections in the joint prosthesis replacement setting. In contrast, linezolid and tedizolid were both inefficient in eradicating bacteria embedded in mature biofilm, confirming the results of a recent study highlighting linezolid MBEC $>1000 \mathrm{mg} / \mathrm{L}$ for a single MSSA reference strain. ${ }^{44}$ Antibiotic tolerance in biofilm is known to rely on various mechanisms: (i) some antibiotics are less efficient against slowly growing bacteria embedded in biofilm; ${ }^{45}$ (ii) biofilm 
structure can act as a physical barrier to antibiotic diffusion; ${ }^{46}$ (iii) persister cells can emerge within biofilms and survive in the presence of high antibiotic concentrations; ${ }^{45}$ and/or (iv) the biofilm-associated lifestyle can modify gene expression, for instance resulting in the induction of the expression of specific efflux pumps. ${ }^{46}$ However, none of these mechanisms has been described for oxazolidinones to date.

Some limitations of this study must be acknowledged. First, cellular pharmacokinetic parameters, including the intracellular final drug concentrations, are unknown and would have been helpful in interpreting the results. Second, antibiotic binding to growth medium proteins (FCS) was not taken into consideration in concentration choices, although it can modify the amount of drug available to diffuse into osteoblasts. However, this parameter is also not considered in the current literature regarding antibiotic bone tissue concentrations, and those selected here are probably the most relevant regarding current pharmacological knowledge. Third, in the present study we tested the activity of linezolid and tedizolid against MSSA isolates. It would have been of interest to test these antibiotics against both MRSA and MSSA. However, MIC frequency distributions of both oxazolidinones are equivalent for MSSA and MRSA isolates. ${ }^{47}$ Moreover, 'real-life' clinical reports showed that linezolid is interchangeably used against MSSA and MRSA isolates in the BJI setting. ${ }^{13,16}$ This is why, in our model, we used MSSA, but based on MIC distributions the results can be transferred to MRSA with the same MIC. Fourth, different exposure times could have been tested, as Nguyen et $a l^{48}$ highlighted differences in antibiotic efficacy between 5, 24 and $72 \mathrm{~h}$ exposure times. Finally, it is well known that biofilm assessment conditions can impact the results. For example, adding plasma to the growth medium in addition to glucose can change biofilm spatial conformation or composition, and then impact the observed MBECs. ${ }^{49}$ However, there are few data in the literature to suggest that one assessment method better mimics in vivo conditions than another.

In conclusion, beyond the well-established effect of oxazolidinones on planktonic S. aureus, linezolid and tedizolid alone do not seem to be optimal candidates to target bacterial phenotypes associated with BJI chronicity. They present strain-dependent intracellular activity, slightly reducing (by 30\%) the intracellular inoculum at bone concentrations. However, they can prevent infection-related cytotoxicity independently of any intracellular bactericidal effect, suggesting a role in modulating the intracellular expression of staphylococcal virulence factors. Although inactive against biofilm-embedded S. aureus, they could prevent biofilm formation, particularly tedizolid, which could be a drug of interest in prevention of biofilm-related orthopaedic infection.

\section{Acknowledgements}

We thank our colleagues from the Institute for Infectious Agents and the 'Pathogénie des Staphylocoques' team, who provided insight and expertise that greatly assisted the research. We are grateful to Michelle Grange for proofreading of this paper prior to submission.

\section{Funding}

This study received the support of an institutional grant from the Hospices Civils de Lyon for 'Young Investigator'.

\section{Transparency declarations}

None to declare.

\section{Supplementary data}

Figure S1 is available as Supplementary data at JAC Online.

\section{References}

1 Lew DP, Waldvogel FA. Osteomyelitis. Lancet 2004; 364: 369-79.

2 Kapadia BH, Berg RA, Daley JA et al. Periprosthetic joint infection. Lancet 2016; 387: 386-94.

3 Ferry T, Perpoint T, Vandenesch F et al. Virulence determinants in Staphylococcus aureus and their involvement in clinical syndromes. Curr Infect Dis Rep 2005; 7: 420-8.

4 Zimmerli W, Sendi P. Orthopaedic biofilm infections. APMIS 2017; 125: 353-64.

5 Osmon DR, Berbari EF, Berendt AR et al. Diagnosis and management of prosthetic joint infection: clinical practice guidelines by the Infectious Diseases Society of America. Clin Infect Dis 2013; 56: e1-25.

6 Jacqueline C, Caillon J. Impact of bacterial biofilm on the treatment of prosthetic joint infections. J Antimicrob Chemother 2014; 69 Suppl 1: i37-40.

7 Valour F, Rasigade J-P, Trouillet-Assant S et al. Delta-toxin production deficiency in Staphylococcus aureus: a diagnostic marker of bone and joint infection chronicity linked with osteoblast invasion and biofilm formation. Clin Microbiol Infect 2015; 21: 568.e1-11.

8 Josse J, Velard F, Gangloff SC. Staphylococcus aureus vs. osteoblast: relationship and consequences in osteomyelitis. Front Cell Infect Microbiol 2015; 5: 85.

9 Valour F, Trouillet-Assant S, Riffard N et al. Antimicrobial activity against intraosteoblastic Staphylococcus aureus. Antimicrob Agents Chemother 2015; 59: 2029-36.

10 Valour F, Karsenty J, Bouaziz A et al. Antimicrobial-related severe adverse events during treatment of bone and joint infection due to methicillinsusceptible Staphylococcus aureus. Antimicrob Agents Chemother 2014; 58: 746-55.

11 Sierra JM, Cabeza JG, Ruiz Chaler M et al. The selection of resistance to and the mutagenicity of different fluoroquinolones in Staphylococcus aureus and Streptococcus pneumoniae. Clin Microbiol Infect 2005; 11: 750-8.

12 O'Neill AJ, Cove JH, Chopra I. Mutation frequencies for resistance to fusidic acid and rifampicin in Staphylococcus aureus. J Antimicrob Chemother 2001; 47: 647-50.

13 Morata L, Senneville E, Bernard L et al. A retrospective review of the clinical experience of linezolid with or without rifampicin in prosthetic joint infections treated with debridement and implant retention. Infect Dis Ther 2014; 3: 235-43.

14 Kutscha-Lissberg F, Hebler U, Muhr G et al. Linezolid penetration into bone and joint tissues infected with methicillin-resistant staphylococci. Antimicrob Agents Chemother 2003; 47: 3964-6.

15 Chen K-H, Huang Y-T, Liao C-H et al. In vitro activities of tedizolid and linezolid against Gram-positive cocci associated with acute bacterial skin and skin structure infections and pneumonia. Antimicrob Agents Chemother 2015; 59: 6262-5.

16 Senneville E, Legout L, Valette $M$ et al. Effectiveness and tolerability of prolonged linezolid treatment for chronic osteomyelitis: a retrospective study. Clin Ther 2006; 28: 1155-63.

17 Metaxas EI, Falagas ME. Update on the safety of linezolid. Expert Opin Drug Saf 2009; 8: 485-91. 
18 Roger C, Roberts JA, Muller L. Clinical pharmacokinetics and pharmacodynamics of oxazolidinones. Clin Pharmacokinet 2018; 57: 559-75.

19 Locke JB, Finn J, Hilgers M et al. Structure-activity relationships of diverse oxazolidinones for linezolid-resistant Staphylococcus aureus strains possessing the cfr methyltransferase gene or ribosomal mutations. Antimicrob Agents Chemother 2010; 54: 5337-43.

20 Ract P, Piau-Couapel C, Compain F et al. In vitro activity of tedizolid and comparator agents against Gram-positive pathogens responsible for bone and joint infections. J Med Microbiol 2017; 66: 1374-8.

21 Rybak JM, Roberts K. Tedizolid phosphate: a next-generation oxazolidinone. Infect Dis Ther 2015; 4: 1-14.

22 Zhanel GG, Love R, Adam Het al. Tedizolid: a novel oxazolidinone with potent activity against multidrug-resistant Gram-positive pathogens. Drugs 2015; 75: 253-70.

23 Livermore DM, Mushtaq S, Warner M et al. Activity of oxazolidinone TR700 against linezolid-susceptible and -resistant staphylococci and enterococci. J Antimicrob Chemother 2009; 63: 713-15.

24 Prokocimer P, De Anda C, Fang E et al. Tedizolid phosphate vs linezolid for treatment of acute bacterial skin and skin structure infections: the ESTABLISH-1 randomized trial. JAMA 2013; 309: 559-69.

25 Flanagan S, McKee EE, Das D et al. Nonclinical and pharmacokinetic assessments to evaluate the potential of tedizolid and linezolid to affect mitochondrial function. Antimicrob Agents Chemother 2015; 59: 178-85.

26 Pautke C, Schieker M, Tischer T et al. Characterization of osteosarcoma cell lines MG-63, Saos-2 and U-2 OS in comparison to human osteoblasts. Anticancer Res 2004; 24: 3743-8.

27 Ong V, Flanagan S, Fang E et al. Absorption, distribution, metabolism, and excretion of the novel antibacterial prodrug tedizolid phosphate. Drug Metab Dispos 2014; 42: 1275-84.

28 Tasse J, Croisier D, Badel-Berchoux $S$ et al. Preliminary results of a new antibiotic susceptibility test against biofilm installation in device-associated infections: the Antibiofilmogram ${ }^{\circledR}$. Pathog Dis 2016; 74: ftw057.

29 Girard LP, Ceri H, Gibb AP et al. MIC versus MBEC to determine the antibiotic sensitivity of Staphylococcus aureus in peritoneal dialysis peritonitis. Perit Dial Int 2010; 30: 652-6.

30 Heydorn A, Nielsen AT, Hentzer M et al. Quantification of biofilm structures by the novel computer program COMSTAT. Microbiology 2000; 146: 2395-407.

31 Ellington JK, Harris M, Hudson MC et al. Intracellular Staphylococcus aureus and antibiotic resistance: implications for treatment of staphylococcal osteomyelitis. J Orthop Res 2006; 24: 87-93.

32 Costerton JW, Stewart PS, Greenberg EP. Bacterial biofilms: a common cause of persistent infections. Science 1999; 284: 1318-22.

33 Mélard A, Garcia LG, Das D et al. Activity of ceftaroline against extracellular (broth) and intracellular (THP-1 monocytes) forms of methicillin-resistant Staphylococcus aureus: comparison with vancomycin, linezolid and daptomycin. J Antimicrob Chemother 2013; 68: 648-58.
34 Garcia LG, Lemaire S, Kahl BC et al. Antibiotic activity against smallcolony variants of Staphylococcus aureus: review of in vitro, animal and clinical data. J Antimicrob Chemother 2013; 68: 1455-64.

35 Hoerr V, Tuchscherr L, Hüve J et al. Bacteria tracking by in vivo magnetic resonance imaging. BMC Biol 2013; 11: 63.

36 Dupieux C, Trouillet-Assant S, Camus C et al. Intraosteoblastic activity of daptomycin in combination with oxacillin and ceftaroline against MSSA and MRSA. J Antimicrob Chemother 2017; 72: 3353-6.

37 Baudoux P, Bles N, Lemaire S et al. Combined effect of pH and concentration on the activities of gentamicin and oxacillin against Staphylococcus aureus in pharmacodynamic models of extracellular and intracellular infections. J Antimicrob Chemother 2007; 59: 246-53.

38 Lemaire S, Tulkens PM, Van Bambeke F. Cellular pharmacokinetics of the novel biaryloxazolidinone radezolid in phagocytic cells: studies with macrophages and polymorphonuclear neutrophils. Antimicrob Agents Chemother 2010; 54: 2540-8.

39 Hodille E, Rose W, Diep BA et al. The role of antibiotics in modulating virulence in Staphylococcus aureus. Clin Microbiol Rev 2017; 30: 887-917.

40 Rasigade J-P, Trouillet-Assant S, Ferry T et al. PSMs of hypervirulent Staphylococcus aureus act as intracellular toxins that kill infected osteoblasts. PLoS One 2013; 8: e63176.

41 Yamaki J, Synold T, Wong-Beringer A. Antivirulence potential of TR-700 and clindamycin on clinical isolates of Staphylococcus aureus producing phenol-soluble modulins. Antimicrob Agents Chemother 2011; 55: 4432-5.

42 Yamaki J, Synold T, Wong-Beringer A. Tigecycline induction of phenolsoluble modulins by invasive methicillin-resistant Staphylococcus aureus strains. Antimicrob Agents Chemother 2013; 57: 4562-5.

43 Otto MP, Martin E, Badiou C et al. Effects of subinhibitory concentrations of antibiotics on virulence factor expression by community-acquired methicillinresistant Staphylococcus aureus. J Antimicrob Chemother 2013; 68: 1524-32.

44 Dall GF, Tsang S-TJ, Gwynne PJ et al. Unexpected synergistic and antagonistic antibiotic activity against Staphylococcus biofilms. J Antimicrob Chemother 2018; 73: 1830-40.

45 Lewis K. Persister cells, dormancy and infectious disease. Nat Rev Microbiol 2007; 5: 48-56.

46 Davies D. Understanding biofilm resistance to antibacterial agents. Nat Rev Drug Discov 2003; 2: 114-22.

47 Karlowsky JA, Hackel MA, Bouchillon SK et al. In vitro activities of tedizolid and comparator antimicrobial agents against clinical isolates of Staphylococcus aureus collected in 12 countries from 2014 to 2016. Diagn Microbiol Infect Dis 2017; 89: 151-7.

48 Nguyen HA, Denis O, Vergison A et al. Intracellular activity of antibiotics in a model of human THP-1 macrophages infected by a Staphylococcus aureus small-colony variant strain isolated from a cystic fibrosis patient: study of antibiotic combinations. Antimicrob Agents Chemother 2009; 53: 1443-9.

49 Zapotoczna M, McCarthy H, Rudkin JK et al. An essential role for coagulase in Staphylococcus aureus biofilm development reveals new therapeutic possibilities for device-related infections. J Infect Dis 2015; 212: 1883-93. 